\title{
Exploiting the Limits of Divergency Scaffolds in Natural Product Synthesis: The Case Study of Sesquiterpenoids
}

\author{
Vera P. Demertzidou, ${ }^{[a]}$ and Alexandros L. Zografos ${ }^{*[a]}$
}

[a] Dr. V. P.Demerzidou, Prof. Dr. A. L. Zografos Laboratory of Organic Chemistry, Department of Chemistry, Aristotle University of Thessaloniki, Main University Campus, 54124, Thessaloniki, Greece. E-mail: alzograf@chem.auth.gr

\begin{abstract}
Divergent synthesis is a powerful but yet underdeveloped method to address the usual drawbacks of poor supply and confined diversity in the total synthesis of natural products. Herein, we describe the rational design behind the selection and the synthesis of a divergency scaffold for sesquiterpenoid lactones synthesis, as a case study, which provides access to a rich collection of carbocycles in different oxidation states within 8,12-sesquiterpenoids through simple, scalable transformations.
\end{abstract}

Secondary metabolites are the main source of drugs and drug leads. ${ }^{[1]}$ By gaining efficient access to their rich chemical diversity and biological properties, pharmaceutical industry can undeniably enter a new era, providing more immediate responses to societal health needs. Towards this goal, divergent synthesis is able to achieve the synthesis of at least part of the natural complexity by relying on the use of the crucial for differentiation common or divergency scaffolds. ${ }^{[2]}$ Ideally, these divergency scaffolds should be easily accessible in multigram quantities, be enantiomerically pure and allow the synthesis of the whole family of natural products that were designed for. Practically though, divergent synthesis suffers from the difficulty to identify and synthesize the optimum divergency scaffold that can lead to the maximum diversity. Focusing on this drawback, our group initiated a campaign, where the main aim is to identify divergency scaffolds of maximum diversity range. ${ }^{[3]}$ As part of this campaign, the divergent synthesis of sesquiterpenoid lactones (SQL), a highly diverse family that plays a pivotal role in the discovery of leads for anticancer drugs has been targeted. ${ }^{[4]}$

The biological properties of SQL are mainly attributed to the highly electrophilic $\alpha$-methylene- $\gamma$-butyrolactone core that they commonly possess. ${ }^{[5]}$ However, besides the well-established connection of SQL with important biological activities, the lability and the complexity of the family restrict the development of scalable, divergent plans to access them. Despite the vast number of reports on selected members, ${ }^{[6]}$ their divergent synthesis is still highly challenging, especially for delivering sufficient quantities for biological screening. Indicative is the fact that even the most successful divergent plans have struggled to achieve extensive divergency in respect of the different oxidation states or regioisomeric forms that the lactone moiety commonly possesses within SQL (Scheme $1 \mathrm{~A}$ ). Adding to that, the highly diverse nature of the family consisting of six different carbocycles, in some cases in both epimeric forms in their junctions, render the divergent synthesis of SQL a challenging task.

Willing to elucidate the way this complexity arises, we turned our attention to their biosynthetic origins. We recognized that despite the preference of most divergent plans for late-stage introduction of the $\alpha$-methylene- $\gamma$-butyrolactone moiety, which is justified by its high lability, Nature does not appear to follow this logic in SQL biosynthesis. Instead, it chooses in most cases to early incorporate lactones as directing components, in order to achieve the selectivity of selected congeners by utilizing the conformational control of allylic strain. ${ }^{[7]}$ Earlier synthetic studies from our group led to the development of a divergency scaffold (compound 1, Scheme 1) able to reach several classes of 8,12 sesquiterpenoids including germacranolides, ${ }^{[3 \mathrm{~b}]}$ guaianolides, ${ }^{[3 \mathrm{c}]}$ cadinanes, ${ }^{[3 \mathrm{c}]}$ and lindenanes. ${ }^{[3 \mathrm{~d}]}$ Despite its undeniable success, the divergent plan was restricted to the introductory oxidative level of furan- 2 and $\alpha, \beta$-unsaturated- $\gamma$-butenolide $\mathbf{3}$ scaffolds, without being able to achieve its expansion to the synthesis of biological intriguing $8,12-\alpha$-methylene- $\gamma$-butyrolactone 4 sesquiterpenoids. What is more, its scalability was poor preventing the access to the larger quantities required for biological screening. To address these drawbacks, the scalable synthesis of a different divergency scaffold, bearing the appropriate oxidation level for the required methylene lactone while retaining the ability to access maximum diversity was envisioned.

In view of the biosynthetic considerations and our earlier results, we considered the synthesis of $\mathbf{5}$ through an early-stage introduction of $\alpha$ methylene- $\gamma$-butyrolactone core, as the plan of choice for accessing 8,12-sesquiterpenoid diversity. Compound 5 was also projected to serve as a divergency scaffold to access our previous routes and also the higher 6-oxidized congeners. The continuously growing family of sesquiterpenoids bearing the syn-8,12-lactone motif (ex. compound 6 in the retrosynthetic analysis) which are largely unexplored by current methods were chosen as initial targets based on their projected potential anticancer activity. ${ }^{[8]}$ Inspired by the concept of pharmacophore- 
directed retrosynthesis, ${ }^{\left[{ }^{[9]}\right.}$ compound $\mathbf{6}$ and its positional 6,12-lactone isomer (not depicted) might be both accessible from 7, through the divergency scaffold $\mathbf{5}$ or by further analysis through $(R)$-carvonic acid $(\mathbf{8})$, by the development of a direct chemoselective syn-lactonization (Scheme 1C). This early-stage introduction of lactone moiety is anticipated not only to ensure the completely stereoselective construction of the remaining tertiary stereocenters in $\mathbf{7}$, but also to produce more biologically oriented scaffolds towards the final goal. ${ }^{[10]}$

A. Indicative diversity in $\underline{8} .12$-sesquiterpenoid lactones
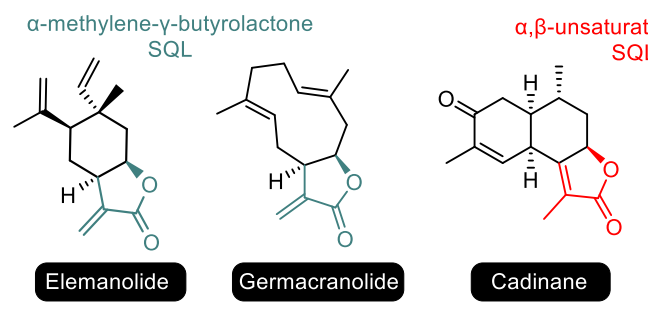

dactone
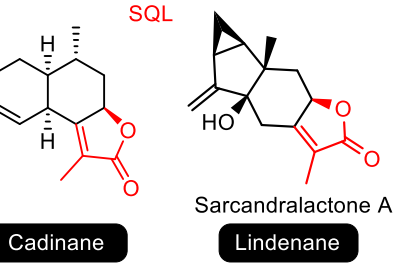

saturated lactone $\alpha$-methylene- $y$-butyrolactone polyoxidized SQL

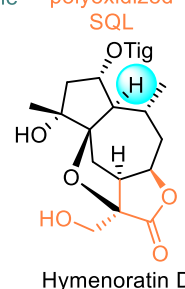

furan SQL

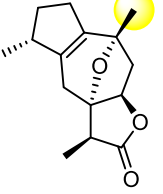

$\mathrm{OAC}$

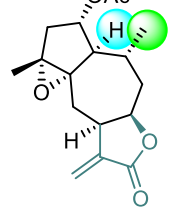

Mexicanin $G$

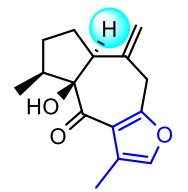

Aquilariaolide

Asteraceae family

B. Current and prior plans<smiles>C#C[C@]1(O)Cc2c(C)coc2C[C@@]1(C)C=C</smiles>

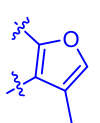

prior work ref.2b-d

no access to a-methylene

Y-butyrolactones SQL)

(R)-carvone

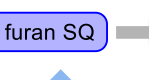

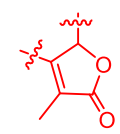

$\alpha, \beta$-unsaturated Y-butenolides SQL this work
Zedoarol

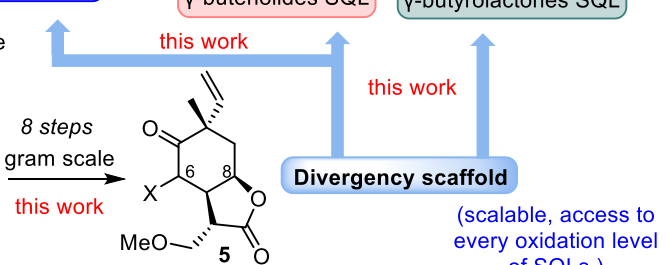

C. Retrosvnthesis . xidation level

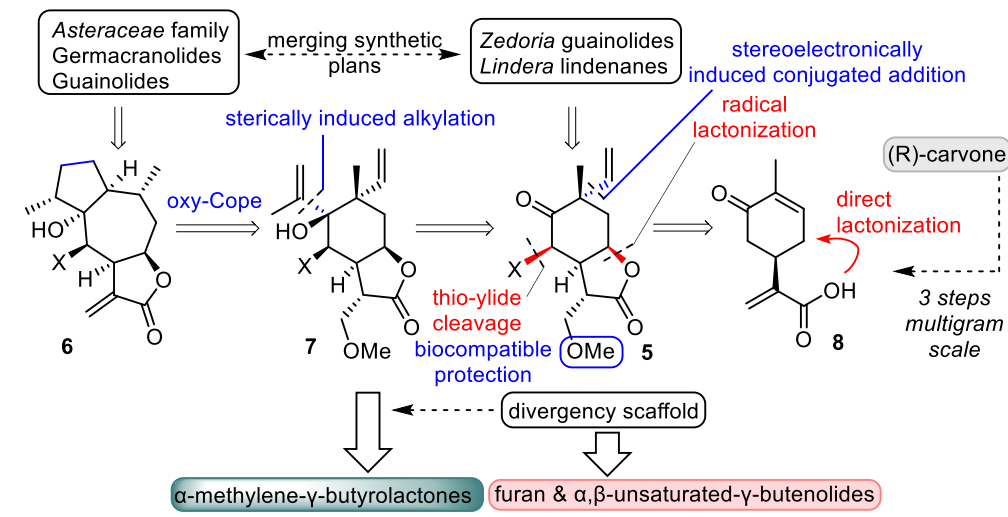

Scheme 1. (A) Selected natural products; (B) Current and prior plans; (C) Retrosynthetic analysis.

The synthesis began with oxidation of $(R)$-carvone to the unstable $(R)$-carvonic acid $(8)$ (Scheme 2). Despite the apparent simplicity of the described transformation, the reported methods were proven unable to deliver reproducible yields of 8 in a multigram scale synthesis $(<10 \%$ overall yield). ${ }^{[11]}$ This early failure prompt us to develop a reliable protocol to access 8 by a novel ene-chlorination of $(R)$-carvone, followed by successive oxidations of the corresponding chloride to 8 . The mixing of $\mathrm{TMSCl}$ and oxone in the presence of $(R)$-carvone provided $75 \%$ yield of $\mathbf{9}$, even at $100 \mathrm{gr}$ scale. ${ }^{[12]}$ A one pot transformation of the crude allylic chloride to acrylic acid 8 followed, using modified conditions of Kornblum and Lindgren protocols to prepare up to $38 \mathrm{~g}$ of $\mathbf{8}$ (38\% overall yield over three steps) in a single batch, without the need for any chromatographic purification. Alternatively, smaller batches were prepared by hydrolyzing allylic chloride with $\mathrm{NaHCO}_{3}$ in aqueous dioxane followed by a combination of a copper (II)/TEMPO catalyzed aerobic oxidation ${ }^{[13]}$ and Lindgren oxidation to provide 8 in $35 \%$ overall yield. 
With multigram quantities of $(R)$-carvonic acid (8) in hand, we next turned our attention to the key syn-8,12-lactonization step. With a rather stabilized enolic form and multiple allylic sites amenable for oxidation in its structure, 8 was found extremely resistant to deliver a chemoselective 8,12-lactonization to 11. Methods utilizing N-halosuccinimides, or halogens under radical conditions, resulted in the formation of polyhalogenized, halolactonized and/or oxidized products, providing only minor amounts of the desired product $\mathbf{1 1}$ (<25\%). Attempts for direct allylic oxidation ${ }^{[14]}$ and utilization of carboxyl as directing group to induce $\mathrm{CH}$-lactonization by known protocols ${ }^{[15-17]}$ failed to provide the desired 8,12-lactone, but instead resulted in some cases in traces of the regioisomeric 6,12-lactone. Considering these facts, we turned our focus on achieving a carboxy-radical directed lactonization. Despite the lack of reported methods, acrylic acids under mild radical conditions were expected to survive extrusion of carbon dioxide, based on their enhanced stability. ${ }^{[18]}$ Indeed, after extensive experimentation, when carvonic acid 8 was allowed to react with excess of PIDA and equimolar amount of iodine under visible light irradiation, a highly chemoselective syn-lactonization was witnessed, affording 11 in $45 \%$ yield (78\% brsm) after a single recrystallization (Scheme 2). Careful monitoring by HNMR evidenced hypervalent iodine conjugates $10 \mathrm{a}-10 \mathrm{~b}$ as the active to homolysis species, allowing the directed hydrogen atom transfer from the most stable allylic position.

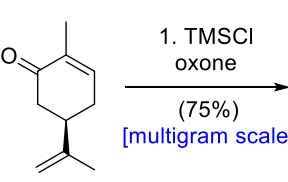

(R)-carvone

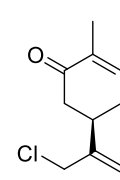

9
2. TMAO $\begin{gathered}\text { DMSO, } \mathrm{CH}_{3} \mathrm{CN} \\ \text { then } \mathrm{NaClO}_{2}\end{gathered}$
$\stackrel{(51 \%),}{\longrightarrow}$
one pot two steps
[multigram scale]

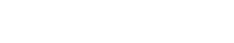

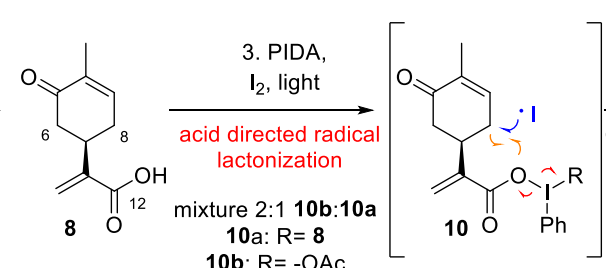

$10 \mathrm{~b}: R=-O A c$
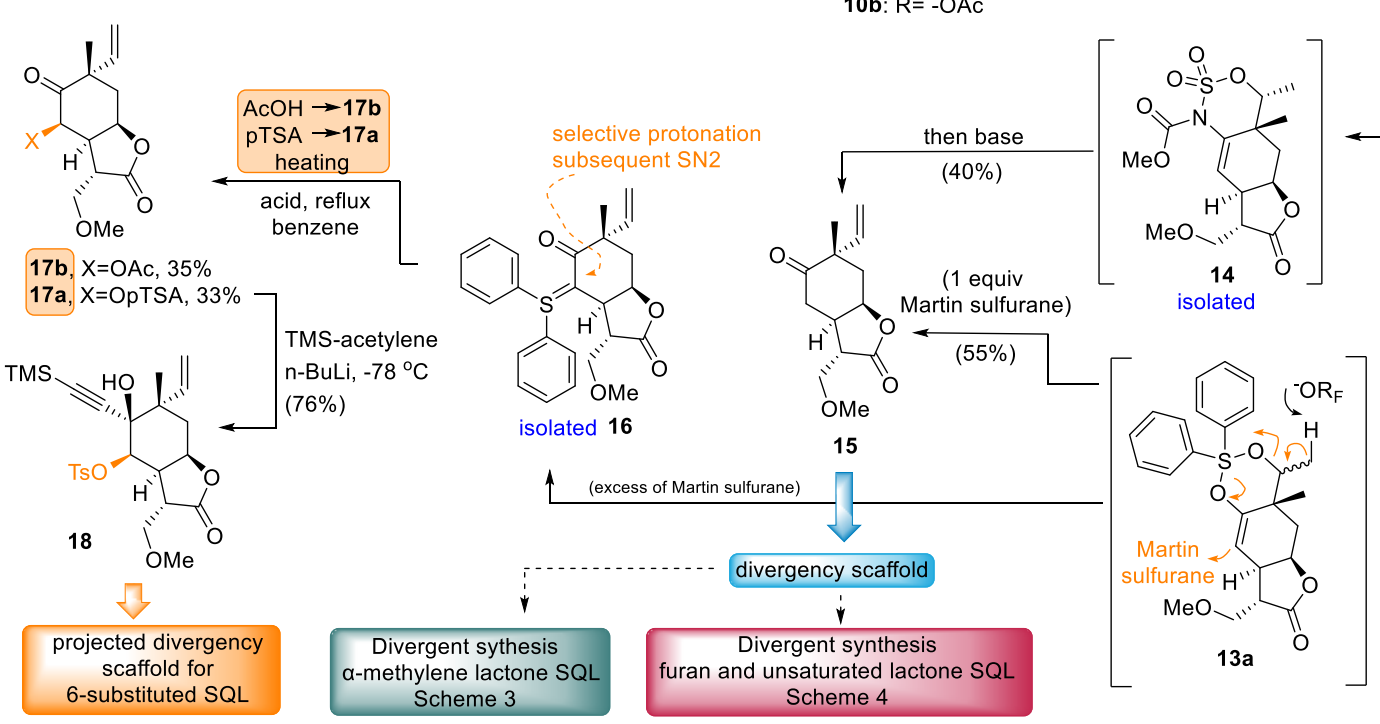

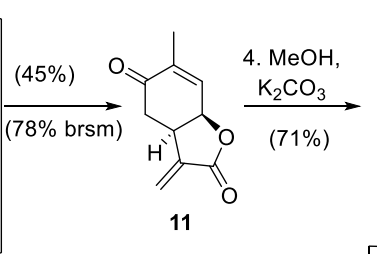

11
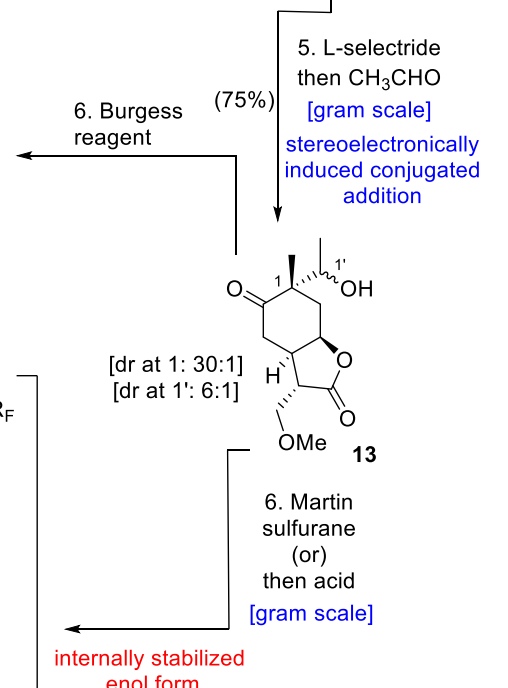

enol form

Scheme 2. Synthetic route to divergency scaffold 15 and its 6-oxidized congeners 18

As expected, the notorious lability of $\alpha$-methylene- $\gamma$-butyrolactone in $\mathbf{1 1}$ towards nucleophiles prohibited its direct alkylation and subsequent introduction of the required tertiary stereocenters. The utilization of a biocompatible protection group was selected. ${ }^{[19]}$ Hence, numerous experiments were run to differentiate the two Michael acceptors in 11 (Scheme 2). Typical conditions of a nucleophile employment (MeOH, or thiophenol) in the presence of $\mathrm{K}_{2} \mathrm{CO}_{3}$ and DMAP failed to provide clean protection of methylene group, due to the interference of the conjugated ketone and/or the cleavage of the lactone moiety. Gratifyingly, addition of DMF as a co-solvent with MeOH in the presence of equimolar amount of $\mathrm{K}_{2} \mathrm{CO}_{3}$ promoted the reaction to completion, affording the desired product 12 in $71 \%$ yield.

Introduction of vinyl group towards 15 followed. The stereoelectronic effects of the syn-8,12-lactone element ensured the selectivity of acetaldehyde addition, which followed the L-selectride conjugated reduction, providing only the desired $1 S$-isomer of 13 (Scheme 2 ). ${ }^{[20]}$ Dehydration by Burgess reagent was unencumbered affording compound 15 in 30\% yield, along with Burgess-substrate hybrid 14 in $20 \%$ yield. The latter was transformed into the desired product by subjection to basic conditions, affording 15 in $40 \%$ overall yield.

Although ketone functionality in $\mathbf{1 5}$ was planned to serve as a reliable enolate precursor for the introduction of the ubiquitous in the SQL family 6 -functionalization, several attempts for its hydroxylation resulted in complex mixture of products, due to the rapid equilibration in the delivered $\alpha$-hydroxy ketone moiety and/or the lactone ring cleavage. Considering these results and focusing on the maximum diversity of our plan, we next sought to internally deliver 6-functionalization through alcohol 13. Accounting on the observation of the Burgess hydrid $\mathbf{1 4}$ formation, we realized that dehydration by Martin sulfurane might drive compound $\mathbf{1 3}$ to deliver sulfur ylides on the 6-position, taking advantage of the internally stabilized intermediate enol form 13a (Scheme 2). Indeed, when excess of Martin sulfurane was applied to 13, 
followed by treatment with pTSA, syn-tosylate analogue 17a (X=O-pTSA) was formed in 33\% yield. The latter can be rationalized by the initial formation of ylide 16 and its subsequent stereoselective protonation-SN2 nucleophilic substitution. ${ }^{[21]}$ Similar results were also obtained when different acids were used instead of pTSA (ex AcOH, product 17b). Compounds 17 can be regarded as projected divergency scaffolds of 6-substituted SQL, after their successful stereoselective alkylation by lithium TMS-acetylene (indicative transformation of 17a provide only one isomer of compound 18). It is interesting to note that Martin sulfurane failed to provide ylides in compounds where alcohol at 1 'position was missing, corroborating to the potential intermediacy of Martin-hybrid 13a for the activation of the 6-position. The use of equimolar amount of Martin sulfurane resulted in the formation of 15 in $55 \%$ yield.

The establishment of the successful route to the desired 15, allowed the plan to be extended to the synthesis of 19 (Scheme 3 ) that serves as divergency scaffold for the synthesis of $\alpha$-methylene- $\gamma$-butyrolactone members, and $\mathbf{3 0}$ which allows the synthesis of furan and $\alpha, \beta$ unsaturated lactone members through 1 (see later Scheme 4).

Introduction of isopropenyl chain solely to the a-face of $\mathbf{1 5}$ towards 19 was crucial for its projected divergency, as early results indicated that epimeric vinyl compound $\mathbf{2 0}$ was unable to initiate the key oxy-Cope reaction, in contrast to our previous results stemming from the utilization of furan analogue 1 (Scheme 3A, inset). Once again, the early introduction of the lactone moiety ensured the stereoselective addition of the isopropenyl chain due to sterics, when cerium 2-propenyl nucleophile was used. Interestingly, addition of harder nucleophiles such as lithium and magnesium 2-propenyl reagents failed to produce useful yields of 19 , due to their preferential attack to the lactone moiety, providing stable lactole products 21 and 22 (Scheme 3B, inset).

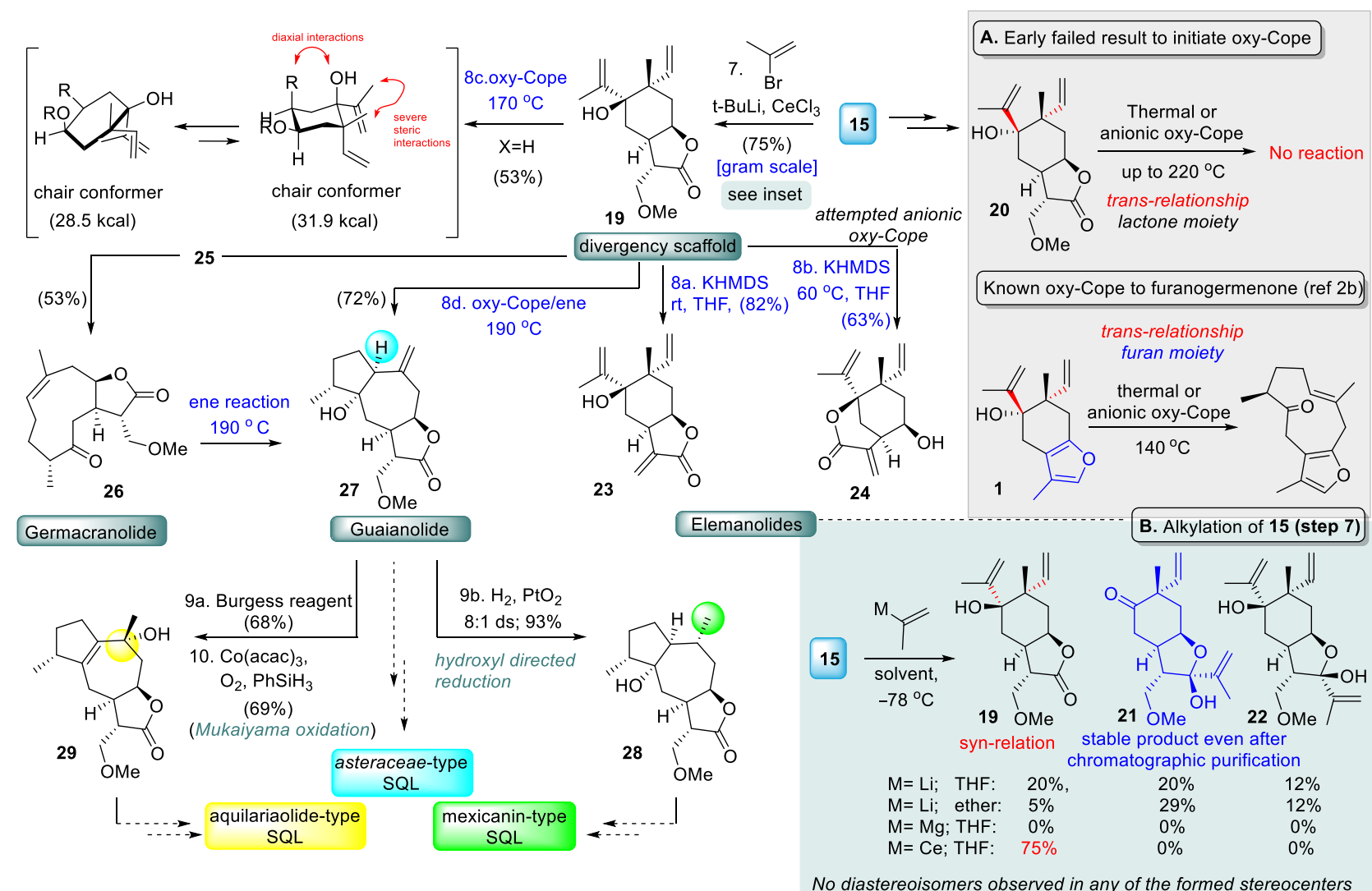

Scheme 3. Divergent synthesis of $\alpha$-methylene SQL and protected congeners.

With a scalable route to 19 in hand, we next tested whether the crucial oxy-Cope reaction could be initiated. Anionic oxy-Cope conditions using KHMDS failed to produce germacranolide 26, but instead provided translactonized product 24 that resembles irregular natural elemanolides (Scheme 3). ${ }^{[22]}$ Employment of lower temperature in the presence of KHMDS easily deprotects methoxy group revealing $\alpha$ methylene-elemanolide 23. Gratifyingly, application of harsh thermal conditions at $170{ }^{\circ} \mathrm{C}$ to 19 transformed it through a highly stereoselective oxy-Cope reaction to methoxy-protected germacranolide 26 in 53\% yield. ${ }^{[23]}$ In addition, heating of 19 at $190{ }^{\circ} \mathrm{C}$ for several hours led to a thermally induced oxy-Cope/ene reaction cascade to deliver guaianolide $\mathbf{2 7}$ in $\mathbf{7 2} \%$ yield, verifying the ability of $\mathbf{1 9}$ to act as a divergency scaffold for the synthesis of syn-8,12-sesquiterpenoids. Identical results were obtained by heating germacranolide 26 at $190{ }^{\circ} \mathrm{C}$, confirming 
its direct involvement in the latter transformation. The trans-1,5- and trans-4,5-relation between the newly formed stereocenters in the enereaction product indicates the boat conformation $\mathbf{2 5}$ as the favourable for the initial oxy-Cope reaction, probably due to the severe methyl interactions for the respective chair conformation. Compound $\mathbf{2 7}$ bears the correct stereochemistry at 1-position to access Asteraceae guaianolides. Catalytic hydrogenation of $\mathbf{2 7}$ by Adam's catalyst provides the stereoselective production of $\alpha$-methyl guaianolide $\mathbf{2 8}$ resembling mexicanin SQLs, ${ }^{[24]}$ while its dehydration by Burgess reagent, followed by Mukaiyama-type alkene hydration leads to compound 29 , a precursor of aquilariaolide SQLs. ${ }^{[25]}$ Both $\mathbf{2 6}$ and $\mathbf{2 7}$ scaffolds can be readily deprotected under basic conditions to regenerate the $\alpha$ methylene- $\gamma$-butyrolactone moiety, and as thus can be regarded as excellent leads for accessing syn-8,12-sesquiterpenoid diversity.

Following the success of $\mathbf{1 9}$ in accessing $\alpha$-methylene- $\gamma$-butyrolactone sesquiterpenoids, we next needed to ascertain the ability of $\mathbf{1 5}$ to further differentiate into furan and $\alpha, \beta$-unsaturated- $\gamma$-butenolide sesquiterpenoids (Scheme 4). To achieve this, 15 must be alkylated by acetylene from the $\beta$-side of the carbonyl group. Attempts to subject $\mathbf{1 5}$ to lithium TMS-acetylene prepared by TMS-acetylene and $n$-BuLi resulted in poor diastereoselectivities for the desired isomer 30 (1:1 ratio over its epimer) (Scheme 4). Contrarily, the use of ethynylmagnesium bromide afforded the desired $\mathbf{3 0}$ in a 3:1 ratio over its epimer, after DBU-promoted methoxy deprotection (Scheme 4). Isomerization of $\alpha$-methylene- $\gamma$-butyrolactone to $\alpha, \beta$-unsaturated- $\gamma$-butenolide $\mathbf{3 1}$ was found difficult to achieve and was only accomplished by the catalytic hydrogen atom transfer to the alkene developed by Shenvi's group. ${ }^{[26]}$ Finally, the direct reduction of the unsaturated moiety to furan by DIBAL furnishes the known furan elemane $1{ }^{[3 \mathrm{~d}]}$ allowing the formal synthesis of furanogermenone, zedoarol, gweicurculactone, curdionolide, epi-lindenene and sarcandralactone A. The formal syntheses were accomplished in the same number of steps as in the previously reported plan (10 steps for common scaffold $\mathbf{1}$ for both synthetic plans), nonetheless with the capacity to deliver gram scale quantities for all synthetic intermediates.

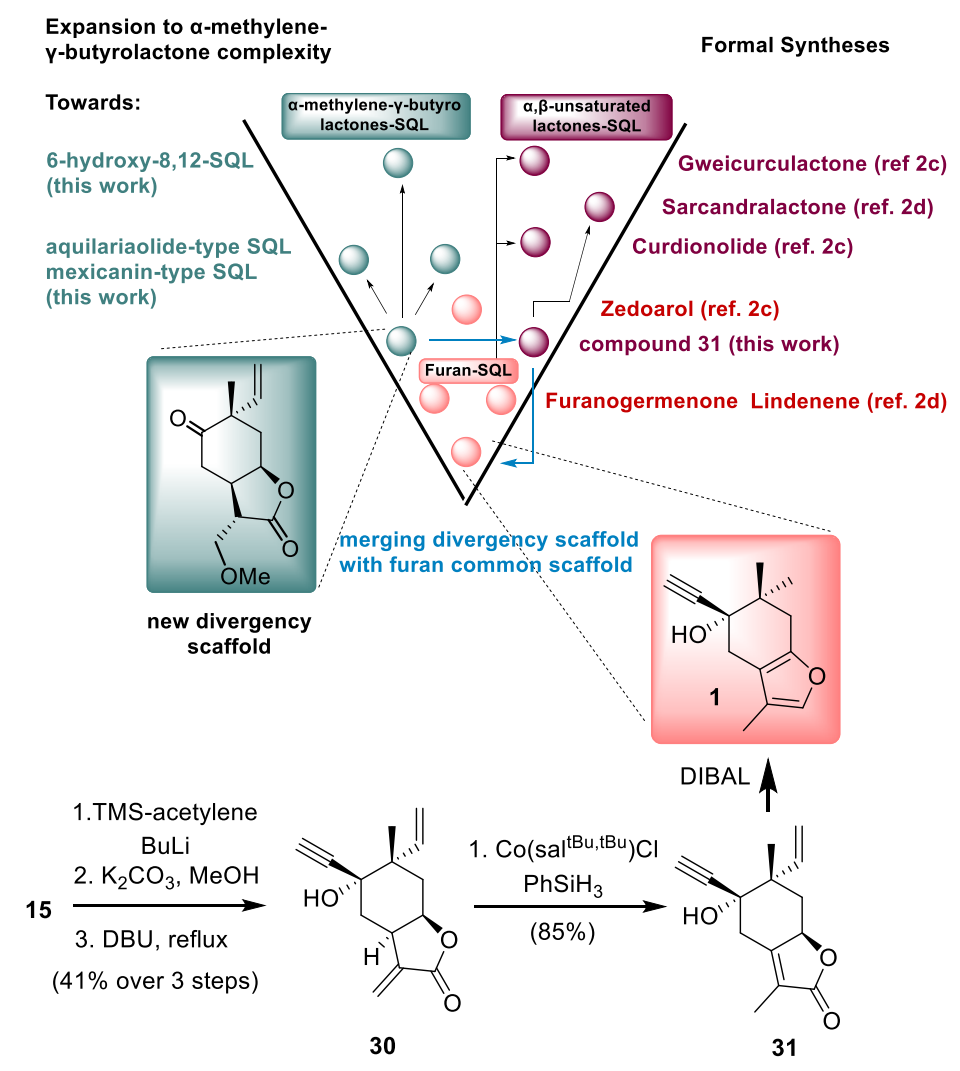

Scheme 4. Merging synthetic routes.

In conclusion, the careful design of appropriate divergency scaffolds renders divergent synthesis a powerful tool for the scalable production of the natural complexity. Our study demonstrates $\mathbf{1 5}$ as a scalable intermediate able to access a rich variety of syn-8,12-SQLs. The route pinpoints the ability of an acrylic carboxylic acid to produce $\alpha$-methylene- $y$-butyrolactone in a single radical step. Additional key steps involve the novel alkoxylation of functionalized elemanes by hydroxyl delivered sulfur ylides and the ene-chlorination of carvone with the aid of TMSCI and oxone. We believe that the described plan will function as a utilizable platform for further development, allowing not only academics, but also medicinal chemists to produce sesquiterpene lactones in gram scale quantities. 


\section{Acknowledgements}

We would like to thank Prof. Shenvi for helpful discussions and for providing us complex Co(sal $\left.{ }^{\mathrm{tButBu}}\right) \mathrm{Cl}$ for the isomerization of 19. We thank ELIDEK agency for scholarship of V.P.D. This work was also supported by the project 'OPENSCREEN-GR' (MIS 5002691) which is implemented under the Action 'Reinforcement of the Research and Innovation Infrastructure', funded by the Operational Program 'Competitiveness, Entrepreneurship and Innovation' (NSRF 2014-2020) and co-financed by Greece and the European Union (European Regional Development Fund). Funding is also provided by Research-Create-Innovate program (project code T1EDK-01161) which is implemented in collaboration with Pharmathen S.A. and co-financed by Greece and the European Union.

\section{References}

[1] D. J. Newman, G. M. Cragg, Natural Products as Sources of New Drugs from 1981 to 2014. J. Nat. Prod. 2016, 79, 629-661.

[2] Seminar report on divergent synthesis by: (a) C.E. Brotherthon, D. L. Boger, Total synthesis of azafluoranthene alkaloids: rufescine and imeluteine. J. Org. Chem. 1984, 49, 4050-4055. For strategies and tactics for the synthesis of divergency scaffolds or common scaffolds can be found on: (a) E. E. Anagnostaki, A. L. Zografos, "Common synthetic scaffolds" in the synthesis of structurally diverse natural products. Chem. Soc. Rev. 2012, 41, 5613-5625. (b) J. Shimokawa, Divergent strategy in natural product total synthesis. Tetrahedron Lett. 2014, 55, 6156-6162. (c) L. Li, Z. Chen, X. Zhang, Y. Jia, Divergent Strategy in Natural Product Synthesis. Chem. Rev. 2018, 118, 3752-3832. (d) A. M. Szpilman, E. M. Carreira, Probing the biology of natural products: molecular editing by diverted total synthesis. Angew. Chem. Int. Ed. 2010, 49, 9592-9628; Angew. Chem. 2010, 122, 9786-9823.

[3] A. D. Fotiadou, A. L. Zografos, Accessing the Structural Diversity of Pyridone Alkaloids: Concise Total Synthesis of Rac-Citridone A. Org. Lett. 2011, 13, 4592-4598. (b) E. E. Anagnostaki, A. L. Zografos, Non-natural Elemane as the "Stepping Stone" for the Synthesis of Germacrane and Guaiane Sesquiterpenes. Org. Lett. 2013, 15, 152-155 (c) E. E. Anagnostaki, V. P. Demertzidou, A. L. Zografos Divergent pathways to furosesquiterpenes: first total syntheses of (+)-zedoarol and (Rac)gweicurculactone. Chem. Commun. 2015, 51, 2364-2367. (d) V. P. Demertzidou, A. L. Zografos, Platinum-catalyzed cycloisomerizations of a common enyne: a divergent entry to cyclopropane sesquiterpenoids. Formal synthesis of sarcandralactone A. Org. Biomol. Chem. 2016, 14, 6942-6946.

[4] (a) S. Zhang, Y.-K. Won, C.-N. Ong, H.-M. Shen, Anti-Cancer Potential of Sesquiterpene Lactones: Bioactivity and Molecular Mechanisms. Current Medicinal Chemistry - Anti-Cancer Agents 2005, 5, 239-249. (b) S. B. Christensen, D. M. Skytte, S. R. Denmeade, C. Dionne, J. V. Moller, P. Nissen, J. T. Isaacs, A Trojan Horse in Drug Development: Targeting of Thapsigargins Towards Prostate Cancer Cells. Anti-Cancer Agents in Medicinal Chemistry 2009, 9, 276-294. (c) I. Nakase, B. Gallis, T. Takatani-Nakase, S. Oh, E. Lacoste, N. P. Singh, D. R. Goodlett, S. Tanaka, S. Futaki, H. Lai, T. Sasaki, Transferrin receptor-dependent cytotoxicity of artemisinin-transferrin conjugates on prostate cancer cells and induction of apoptosis. Cancer Letters 2009, 274, 290-298. (d) A. Ghantous, H. Gali-Muhtasib, H. Vuorela, N. A. Saliba, N. Darwiche, What made sesquiterpene lactones reach cancer clinical trials? Drug Discovery Today 2010, 15, 668-678. (e) M. R. Kreuger, S. Grootjans, M. W. Biavatti, P. Vandenabeele, K. D'Herde, Sesquiterpene lactones as drugs with multiple targets in cancer treatment: focus on parthenolide. Anticancer drugs 2012, 23, 883-896. (f) M. Chadwick, H. Trewin, F. Gawthrop, C. Wagstaff, Sesquiterpenoid Lactones: Benefits to plants and people. Int. J. Mol. Sci. 2013, 14, 12780-12805. (g) Y. Ren, J. Yu, A. D. Kinghorn, Development of Anticancer Agents from Plant-derived Sesquiterpene Lactones. Curr. Med. Chem. 2016, 23, 2397-2420.

[5] (a) D. K. Black, The addition of L-cysteine to unsaturated lactones and related compounds. J. Chem. Soc. C 1966, 1123-1127. (b) J. B. Jones, J. M. Young, Carcinogenicity of lactones. III. The reactions of unsaturated .gamma.-lactones with L-cysteine. J. Med. Chem. 1968, 11, 1176-1182. (c) S. M. Kupchan, D. C. Fessler, M. A. Eakin, T. J. Giacobbe, Reactions of Alpha Methylene Lactone Tumor Inhibitors with Model Biological Nucleophiles. Science 1970, 168, 376-378. (d) S. Amslinger, The Tunable Functionality of $\alpha, \beta$-Unsaturated Carbonyl Compounds Enables Their Differential Application in Biological Systems. ChemMedChem 2010, 5, 351-356. (e) K. Camara, S. S. Kamat, C. C. Lasota, B. F. Cravatt, A. R. Howell, Combining cross-metathesis and activity-based protein profiling: New $\beta$ lactone motifs for targeting serine hydrolases. Bioorg. Med. Chem. Lett. 2015, 25, 317-325.

[6] For selected divergent syntheses of sesquiterpene lactones, see the following: (a) S. F. Oliver, K. Högenauer, O. Simic, A. Antonello, M. D. Smith, S. V. Ley, A Route to the Thapsigargins from (S)-Carvone Providing a Substrate-Controlled Total Synthesis of Trilobolide, Nortrilobolide, and Thapsivillosin F. Angew. Chem. Int. Ed. 2003, 42, 5996-6000; Angew. Chem. 2003, 115, 6178-6182; (b) G. Valot, J. Garcia, V. Duplan, C. Serba, S. Barluenga, N. Winssinger, Diversity-oriented synthesis of diverse polycyclic scaffolds inspired by the logic of sesquiterpene lactones biosynthesis. Angew. Chem. Int. Ed. 2012, 51, 5391-5394; Angew. Chem. 2012, 122, 5487-5490; (c) C. Li, T. Dong, Q. Li, X. Lei, Probing the anticancer mechanism of (-)-ainsliatrimer A through diverted total synthesis and biorthogonal ligation. Angew. Chem. Int. Ed. 2014, 53, 12111-11115; Angew. Chem. 2014, 126, 12307-12311; (d) W.-B. Sun, X. Wang, B.-F. Sun, J.-P. Zou, G.-Q. Lin, Catalytic Asymmetric Total Synthesis of Hedyosumins A, B, and C. Org. Lett. 2016, 18, 1219-1221; (e) X. Wang, W.-B. Sun, J.-P. Zou, G.-Q. Lin, B.-F Sun, Asymmetric total synthesis of hedyosumin E aglycon, 7,10-epoxyhedyosminolide and ent-zedolactone A. Org. Biomol. Chem. 2016, 14, 10581-10584; (f) R. Lagouette, C. Serba, D. Abegg, D. G. Hoch, A. Adibekian, N. Winssinger, Divergent synthesis and identification of the cellular targets of deoxyelephantopins. Nat. Commun. 2016, 7, 12470; (g) X. Hu, S. Xu, T. J. Maimone, A Double Allylation Strategy for Gram-Scale Guaianolide Production: Total Synthesis of (+)-Mikanokryptin. Angew. Chem. Int. Ed. 2017, 56, 1624-1628; Angew. Chem. 2017, 129, 1646-1650; (h) H. Chu, J. M. Smith, J. Felding, P. S. Baran, Scalable synthesis of (-)-thapsigargin. ACS Cent. Sci. 2017, 3, 47-51; (i) D. Chen, A. P. Evans, A concice, efficient and scalable total synthesis of thapsigargin and nortrilobolide from (R)-(-)-carvone. J. Am. Chem. Soc. 2017, 139, 6046-6049; (j) X. Hu, A. J. Musacchio, X. Shen, Y. Tao, T. J. Maimone, Allylative Approaches to the Synthesis of Complex Guaianolide Sesquiterpenes from Apiaceae and Asteraceae. J. Am. Chem. Soc. 2019, 141, 14904-14915; (k) B. Du, Z. Huang, X. Wang, T. Chen, G. Shen, S. Fu, B. Liu, A unified strategy toward total synthesis of lindenane sesquiterpenoid [4+2] dimers. Nat. Commun. 2019, $10,1892$.

[7] Please refer to the SI for postulated correlation of early stage lactonization with observed selectivity on selected sesquiterpenoid lactones.

[8] (a) A. C. Beekman, H. J. Woerdenbag, W. van Uden, N. Pras, A. W. T. Konings, H. V. Wikström, T. J. Schmidt, Structure-Cytotoxicity Relationships of Some Helenanolide-Type Sesquiterpene Lactones. J. Nat. Prod. 1997, 60, 252-257. (b) M. T. Scotti, M. B. Fernandes, M. J. P. Ferreira, V. P. Emerenciano, Quantitative structure-activity relationship of sesquiterpene lactones with cytotoxic activity. Bioorg. Med. Chem. 2007, 15, $2927-2934$. 
[9] (a) D. Romo, Bioactivity-guided retrosynthesis: "Upping the ante" for natural product synthesis. Planta Med. 2015, 81; (b) J. R. Hudlicky, G. A. Sulikowski, Simple start for complex products. Nat. Chem. 2019, 11, 297-298; (c) M. E. Abbasov, R. Alvarino, C. M. Chaheine, E. Alonso, J. A. Sanchez, M. L. Conner, A. Alfonso, M. Jaspars, L. M. Botana, D. Romo, Simplified immunosuppressive and neuroprotective agents based on gracillin A. Nat. Chem. 2019, 11, 342-350.

[10] See supporting information for analysis of conformational models and for the synthesis and the presentation of the stereochemical outcome in the absence of lactone formation.

[11] (a) W. Engel, In Vivo Studies on the Metabolism of the Monoterpenes $S-(+)-$ and $R$-(-)-Carvone in Humans Using the Metabolism of Ingestion-Correlated Amounts (MICA) Approach. J. Agric. Food Chem. 2001, 49, 4069-4075. (b) D. W. Lee, C. F. Manful, J. R. Gone, Y. Ma, W. A. Donaldson, Reactivity of acyclic (pentadienyl)iron(1+) cations with phosphonate stabilized nucleophiles: application to the synthesis of oxygenated metabolites of carvone. Tetrahedron 2016, 72 , 753-759.

[12] See the supporting information for comparison of allylic chlorination methods and the optimization of TMSCl, oxone protocol.

[13] Modified conditions of the provided references are used: (a) X. Jiang, J. Zhang, S. Ma, Iron Catalysis for Room-Temperature Aerobic Oxidation of Alcohols to Carboxylic Acids. J. Am. Chem. Soc. 2016, 138, 8344-8347. (b) D. Könning, T. Olbrisch, F. D. Sypaseuth, C. C. Tzschucke, M. Christmann, Oxidation of allylic and benzylic alcohols to aldehydes and carboxylic acids. Chem. Commun. 2014, 50, 5014-5016.

[14] M. S. Chen, M. C. White, A Sulfoxide-Promoted, Catalytic Method for the Regioselective Synthesis of Allylic Acetates from Monosubstituted Olefins via C-H oxidation. J. Am. Chem. Soc. 2004, 126, 1346-1347.

[15] M. Yang, X. Jiang, W.-J. Shi, Q.-L. Zhu, Z.-J. Shi, Direct Lactonization of 2-Arylacetic Acids through Pd(II)-Catalyzed C-H Activation/C-O Formation. Org. Lett. 2013, 15, 690-693.

[16] S. Sathyamoorthi, J. Du Bois, Copper-Catalyzed Oxidative Cyclization of Carboxylic Acids. Org. Lett. 2016, 18, 6308-6311.

[17] M. P. DeMartino, K. Chen, P. S. Baran, Intermolecular Enolate Heterocoupling: Scope, Mechanism, and Application. J. Am. Chem. Soc. 2008, 130, 11546-11560.

[18] (a) D. H. R. Barton, D. Crich, W. B. Motherwell, New and improved methods for the radical decarboxylation of acids. J. Chem. Soc., Chem. Commun. 1983, 939941. (b) D. H. R. Barton, D. Crich, W. B. Motherwell, A practical alternative to the hunsdiecker reaction. Tetrahedron Lett. 1983, $24,4979-4982$.

[19] Methoxy-protected butenolides can be readily cleaved in cells, in accordance to similar results for amino-protected sesquiterpenoids: Woods, J. R.; Mo, H.; Bieberich, A. A..; Alavanja, T.; Colby, D. A. Amino-derivatives of the sesquiterpene lactone class of natural products as prodrugs. Med. Chem. Commun. 2013, 4, 27-33.

[20] (S)-Isomer on the alcohol is favored in a ratio of 6:1 as predicted by Zimmerman-Traxler model. Note that the conjugated reduction of ( $S$ )-carvone under identical conditions provided the non-desired $1 R$-isomer in a ratio of 9:1.

[21] For similar transformation please see: (a) X. Huang, R. Goddard, N. Maulide, A Direct Ylide Transfer to Carbonyl Derivatives and Heteroaromatic Compounds. Angew. Chem. Int. Ed. 2010, 49, 8979-8983; Angew. Chem. 2010, 122, 9163-9167; (b) X. Huang, M. Patil, C. Fares, W. Thiel, N. Maulide, Sulful (IV)-mediated transformations: From ylide transfer to metal free arylation of carbonyl compounds. J. Am. Chem. Soc. 2013, 135, 7312-7323.

[22] B. P. Bashyal, S. P. McLaughlin, A. A. L. Gunatilaka, Zinagrandinolides A-C, Cytotoxic ס-Elemanolide-Type Sesquiterpene Lactones from Zinnia grandiflora. J. Nat. Prod. 2006, 69, 1820-1822.

[23] C. Zdero, F. Bohlmann, R. M. King, H. Robinson, The first 12.8ß-germacranolide and other constituents from bolivian Stevia species. Phytochemistry 1988, 27, 2835-2842.

[24] A. Romo de Vivar, G. Delgado, E. Huerta, Structure and stereochemistry of mexicanin G, an intermediate in the biogenesis of helenanolides. Phytochemistry 1985, 24, 2977-2979.

[25] C.-N. Mi, W.-L. Mei, H. Wang, L. Yang, W.-H. Dong, C.-J. Gai, J.-Z. Yuan, W.-Z. Long, H.-F. Dai, Filoterapia 2019, $135,79-84$.

[26] S. W. M. Crossley, F. Barabé, R. A. Shenvi, J. Am. Chem. Soc. 2014, 136, 16788-16791. 
Divergent Synthesis of 8,12-sesquiterpenoid lactones

Pharmacophore-directed
retrosynthesis

- Multigram scale

thio-ylide

cleavage

biocompatible
protection

$$
\text { a-methylene- } \gamma \text {-butenolides }
$$

furan \& $\alpha, \beta$-unsaturated- $\gamma$-butenolides 